${ }^{\odot}$ Entomologica Fennica. 3 July 2002

\title{
New records of Corixidae (Heteroptera) from northeastern USA and eastern Canada, with one new synonymy
}

\author{
Antti Jansson
}

\begin{abstract}
Jansson, A. 2002: New records of Corixidae (Heteroptera) from northeastern USA and eastern Canada, with one new synonymy. - Entomol. Fennica 13: 85-88.
\end{abstract}

The following numbers of new state and province records of Corixidae are reported: Maine 18, New Hampshire 27, Vermont 32, Massachusetts 11, Connecticut 23, Rhode Island 22, New York 12, Ontario 9, Quebec 4, New Brunswick 3, Nova Scotia 22, Prince Edward Island 6, Newfoundland 2, and Labrador 7. Trichocorixa verticalis fenestrata Walley is synonymised with the nominate subspecies.

Antti Jansson, Kukinkuja 4 E 61, FIN-01620 Vantaa, Finland; E-mail: antti.jansson@helsinki.fi (Research Associate of the Zoological Museum, University of Helsinki.)

Received 6 September 2000, accepted 20 May 2001

\section{Introduction}

The northeastern United States and parts of eastern Canada have been badly neglected areas in distributional records of Corixidae. In his revision of the Corixidae of the Western Hemisphere, Hungerford (1948, including Sailer 1948) listed the following numbers of species for northeastern USA: Maine 16, New Hampshire 20, Vermont 3, Massachusetts 28, Connecticut 19, Rhode Island 15, and New York 35. Polhemus et al. (1988) added three species to Maine, one to New Hampshire, one to Massachusetts, five to Connecticut, and one to Rhode Island. For eastern Canada, Hungerford (1948) reported the following numbers of species per province: Ontario 22, Quebec 19, New Brunswick 7, Nova Scotia 4, Prince Edward Island none, Newfoundland 9, and Labrador 2. Note that Hungerford considered the latter two as separate areas, and because Labrador is biologically very different from the Island of Newfoundland, the present paper follows Hungerford's concept. Lansbury (1955, including also some earlier publications) added new provincial records as follows: Ontario 4, Quebec 9, New Brunswick 1, Newfoundland 3, and Labrador 3. Larsen and Colbo (1983, overlooking Lansbury 1955) added eight species to the list for the Island of Newfoundland. Polhemus et al. (1988) added new provincial records for eastern Canada as follows: Ontario 17, Quebec 13, New Brunswick 15, Nova Scotia 10, Prince Edward Island 10, and Newfoundland 1 . I, too, have exactly matching data from specimens that I identified in the early 1980's mainly for the Canadian National collection. I asked John T. Polhemus about the origins of these records, but there are seemingly no notes concerning them. Therefore, there is a possibility to have missed some essential publications.

\section{Distribution}

While preparing a revision of North American Corixidae, I had an opportunity to study several other North American collections and thus, as a preliminary report, I can now list a number of new records for northeastern USA and eastern Canada (Table 1). 
Table 1. The species of Corixidae recorded from northeastern USA and eastern Canada: ME, NH, VT, MA, CT, RI, NY, ONT, QUE, NB, NS, PEI, NFL and LB. Symbols: $X=$ previous record of the species, $X^{\star}=$ new state or province record, $-=$ species not recorded so far for the state or province in question.

\begin{tabular}{|c|c|c|c|c|c|c|c|c|c|c|c|c|c|c|}
\hline Species & ME & $\mathrm{NH}$ & VT & MA & CT & $\mathrm{RI}$ & NY & ONT & QUE & NB & NS & PEI & NFL & LB \\
\hline Cymatia americana Hussey & - & - & - & - & - & - & - & $x$ & $x$ & - & - & - & - & - \\
\hline Dasycorixa hybrida (Hungerford) ${ }^{1}$ & - & - & - & - & - & - & - & $X^{*}$ & $X^{*}$ & - & - & - & $\mathrm{X}^{\star 2}$ & - \\
\hline D. johanseni (Walley) ${ }^{1}$ & - & - & - & - & - & - & - & - & $\mathrm{x}$ & - & - & - & - & - \\
\hline Glaenocorisa cavifrons (Thomson) ${ }^{1,3}$ & - & - & - & - & - & - & - & - & $x$ & - & - & - & $\mathrm{X}$ & $X^{*}$ \\
\hline Arctocorisa chanceae Hungerford & - & - & - & - & - & - & - & $X^{*}$ & $x$ & - & - & - & $x$ & $X^{*}$ \\
\hline A. convexa (Fieber) & - & - & - & - & - & - & - & $X^{*}$ & $x$ & - & - & - & $\mathrm{x}$ & $x$ \\
\hline A. planifrons (Kirby) & - & - & - & - & - & - & - & - & - & - & - & - & $x$ & $x$ \\
\hline Callicorixa alaskensis Hungerford & $X^{*}$ & $x$ & $X^{*}$ & - & $X^{*}$ & - & $\mathrm{X}$ & $\mathrm{X}$ & $x$ & $x$ & $x$ & - & $x$ & $x$ \\
\hline C. audeni Hungerford & $x$ & $X^{*}$ & $X^{*}$ & $X^{*}$ & $X^{*}$ & $X^{*}$ & $X^{*}$ & $x$ & $x$ & $\mathrm{X}$ & $x$ & - & $x$ & $X^{*}$ \\
\hline Cenocorixa utahensis $(\mathrm{Hu}$ & - & - & - & - & - & - & - & $\mathrm{x}$ & - & - & - & - & - & - \\
\hline Corisella edulis (Champion) & - & $\mathrm{X}^{*}$ & - & - & - & - & - & - & - & - & - & - & - & - \\
\hline C. tarsalis (Fieber) & - & - & - & $X^{*}$ & $X^{*}$ & - & $X^{4}$ & $\mathrm{x}$ & - & - & - & - & - & - \\
\hline Hesperocorixa atopodo & $X$ & $\mathrm{X}$ & $X^{*}$ & $\mathrm{X}$ & $\mathrm{X}$ & $X$ & $\mathrm{x}$ & $\mathrm{X}$ & $x$ & $X$ & $x$ & $\mathrm{X}$ & $X$ & - \\
\hline H. harrisii (Uhler) ${ }^{5}$ & - & - & $x$ & $x$ & $X^{*}$ & $X$ & $x$ & $x$ & $x$ & - & $x$ & $\mathrm{X}$ & - & - \\
\hline H. interrupta (Say) & $\mathrm{X}$ & $\mathrm{X}$ & $X$ & $\mathrm{X}$ & $\mathrm{X}$ & $\mathrm{X}$ & $\mathrm{X}$ & $\mathrm{X}$ & $x$ & $\mathrm{X}^{*}$ & $\mathrm{X}^{\star}$ & - & - & - \\
\hline H. kennicottii (Uhler) $)^{5}$ & $x$ & $x$ & $X^{*}$ & $x$ & $x$ & $X^{*}$ & $x$ & $x$ & $x$ & - & $\mathrm{X}^{*}$ & - & - & - \\
\hline H. Iobata (Hungerford) & $\mathrm{X}$ & $x$ & $X^{*}$ & $x$ & $X^{*}$ & $x$ & $x$ & - & - & - & $\mathrm{X}^{*}$ & - & - & - \\
\hline H. lucida (Abbot & - & $X^{*}$ & $X^{*}$ & $x$ & $x$ & $x$ & - & - & - & - & - & - & - & - \\
\hline H. martini (Walley) & - & - & - & - & $X^{*}$ & $X^{*}$ & - & - & - & - & - & - & - & - \\
\hline H. michiganensis (Hungerford) & $X^{*}$ & $x$ & $X^{*}$ & $x$ & - & - & $x$ & $x$ & $x$ & $\mathrm{X}$ & $X^{*}$ & - & - & - \\
\hline H. minor (Abbott) & - & $X^{*}$ & - & $X$ & $X^{*}$ & $x$ & $X^{4}$ & - & - & - & - & - & - & - \\
\hline H. minorella (Hungerford) & $x$ & $x$ & - & $X^{*}$ & $x$ & - & $X^{*}$ & $\mathrm{X}$ & $x$ & $\mathrm{X}$ & $x$ & $X^{*}$ & $x$ & - \\
\hline H. nitida (Fieber & $x$ & $\mathrm{X}^{*}$ & - & $x$ & $x$ & $\mathrm{x}$ & $\mathrm{x}$ & - & - & - & - & - & - & - \\
\hline H. obliqua (Hungerford) & - & - & $X^{*}$ & $x$ & $X^{*}$ & $X^{*}$ & $\mathrm{x}$ & $X^{*}$ & - & - & - & - & - & - \\
\hline H. scabricula (Walley) & - & $X^{*}$ & $X^{*}$ & $X^{*}$ & $X^{*}$ & $X^{*}$ & - & $x$ & $x$ & $x$ & $\mathrm{X}^{*}$ & $\mathrm{X}$ & - & - \\
\hline H. semilucida ( & - & $X^{*}$ & $X^{*}$ & $x$ & $X^{*}$ & - & $x$ & $\mathrm{X}$ & $X^{*}$ & - & $X^{*}$ & - & - & - \\
\hline H. vulgaris (Hun & $x$ & $\mathrm{x}$ & $\mathrm{X}$ & $x$ & $\mathrm{x}$ & $\mathrm{X}$ & $\mathrm{x}$ & $\mathrm{X}$ & $x$ & $x$ & $\mathrm{X}^{*}$ & - & - & - \\
\hline Palmacorixa buenoi A & $\mathrm{X}^{\star}$ & $\mathrm{X}^{*}$ & - & $x$ & $X^{*}$ & $\mathrm{X}^{\star}$ & $\mathrm{X}$ & $\mathrm{X}$ & $x$ & - & $\mathrm{X}^{\star}$ & - & $\mathrm{X}$ & - \\
\hline P. gillettei Abbott & $\mathrm{X}^{*}$ & - & $X^{*}$ & - & - & - & - & $x$ & $x$ & - & - & - & - & - \\
\hline P. nana nana Walley & - & - & - & - & - & - & $x$ & $\mathrm{X}^{*}$ & $x$ & - & - & - & - & - \\
\hline Ramphocorixa acuminata (Uhler) & - & - & - & - & - & - & - & $X^{*}$ & - & - & - & - & - & - \\
\hline Sigara alternata (Say) & $X^{*}$ & $\mathrm{X}$ & $X^{*}$ & $x$ & $x$ & $x$ & $x$ & $\mathrm{x}$ & $x$ & $\mathrm{X}$ & $x$ & $x$ & $X^{*}$ & - \\
\hline S. bicoloripennis (Walley) & $X^{*}$ & $X^{*}$ & $X^{*}$ & - & - & $X^{*}$ & $X^{*}$ & $\mathrm{X}$ & $x$ & $x$ & $\mathrm{X}^{*}$ & $X^{*}$ & $X$ & - \\
\hline S. compressoidea (Hungerford) & $x$ & $X^{*}$ & $X^{*}$ & $x$ & $x$ & $X^{*}$ & $x$ & $\mathrm{x}$ & $x$ & $\mathrm{X}$ & $x$ & - & $\mathrm{X}$ & - \\
\hline ephala (Hungerford) & - & $X^{*}$ & - & - & - & - & - & $\mathrm{x}$ & $x$ & - & $x$ & $\mathrm{x}$ & $X$ & - \\
\hline S. decol & $x$ & $X^{*}$ & $X^{*}$ & $x$ & $x$ & $X^{*}$ & $X^{*}$ & $X^{*}$ & - & - & - & - & - & - \\
\hline S. decoratella (Hungerf & $x$ & $\mathrm{X}^{*}$ & $X^{*}$ & $x$ & $x$ & $x$ & $x$ & $x$ & $x$ & $\mathrm{X}$ & $x$ & $\mathrm{X}$ & $\mathrm{X}$ & $x$ \\
\hline S. defecta Hungerford \& Sailer & - & - & - & - & - & -6 & - & $x$ & $x$ & - & $x$ & - & - & - \\
\hline hro Hungroro & - & $X^{*}$ & $X^{*}$ & $X^{*}$ & $X^{*}$ & - & $x$ & $\mathrm{x}$ & - & - & $x$ & - & - & - \\
\hline S. douglase & $X^{*}$ & $X^{*}$ & $X^{*}$ & $x^{6}$ & $x$ & $X^{*}$ & $x$ & $\mathrm{X}^{*}$ & $x$ & - & $x$ & $\mathrm{X}^{*}$ & - & - \\
\hline S. fallenoidea (Hungerford) & - & - & - & - & - & - & $X^{*}$ & - & - & - & - & - & - & - \\
\hline S. gordita (Abbott) & $X^{*}$ & $X^{*}$ & - & $x$ & $X^{*}$ & $X^{*}$ & $X^{\star 7}$ & - & - & - & - & - & - & - \\
\hline S. grossolineata Hun & $x$ & $x$ & $X^{*}$ & $x$ & $X^{*}$ & $X^{*}$ & $x$ & $x$ & $x$ & $x$ & $X^{*}$ & - & - & - \\
\hline S. hubbelli (Hungerford) & - & $x$ & - & - & $X^{*}$ & - & - & $x$ & - & - & - & - & - & - \\
\hline S. johnstoni Hungerford & $X^{*}$ & $X^{*}$ & - & $X^{*}$ & $X^{*}$ & $X^{*}$ & $X^{*}$ & $\mathrm{X}$ & $x$ & $x$ & $X^{*}$ & - & - & - \\
\hline S. knighti Hungerford & $\mathrm{X}^{*}$ & _- & $\mathrm{X}^{*}$ & - & - & - & _- & $\mathrm{x}$ & $x$ & $\mathrm{X}$ & $x$ & - & - & - \\
\hline S. lineata (Forster) & - & - & - & - & $X^{*}$ & - & - & $x$ & $x$ & - & $\mathrm{X}^{*}$ & - & - & - \\
\hline S. mackinacensis $(\mathrm{Hu}$ & $X^{*}$ & $x$ & - & $\mathrm{X}^{*}$ & $x$ & $X^{*}$ & $X^{*}$ & $x$ & $x$ & $x$ & $\mathrm{X}^{*}$ & - & - & - \\
\hline S. macropala (Hungerford) & $x$ & $\mathrm{X}^{*}$ & - & $x$ & $X^{*}$ & $x$ & $x$ & - & $X^{*}$ & $\mathrm{X}^{*}$ & $\mathrm{X}^{*}$ & - & $X^{8}$ & - \\
\hline S. mathesoni Hungerford & $\mathrm{X}^{*}$ & $X^{*}$ & - & $X^{*}$ & $x$ & - & $x$ & $x$ & - & - & $x$ & $X^{*}$ & $\mathrm{x}$ & - \\
\hline S. modesta (Abbott) & $X^{*}$ & $x$ & $X^{*}$ & $x$ & $x$ & $X^{*}$ & $x$ & $x$ & $X^{*}$ & - & - & - & - & - \\
\hline
\end{tabular}


Table 1. Continued.

\begin{tabular}{|c|c|c|c|c|c|c|c|c|c|c|c|c|c|c|}
\hline Species & ME & $\mathrm{NH}$ & VT & MA & CT & $\mathrm{RI}$ & NY & ONT & QUE & NB & NS & PEI & NFL & LB \\
\hline S. mullettensis (Hungerford) & $\mathrm{X}$ & $x$ & $X^{*}$ & - & $X$ & $X$ & $x$ & $x$ & $x$ & $x$ & $X^{*}$ & - & - & - \\
\hline S. ornata (Abbott) & $x$ & $X^{*}$ & $X^{*}$ & $x$ & $X$ & $x$ & $x$ & $x$ & $x$ & $x$ & - & - & - & - \\
\hline S. penniensis (Hungerford) & $\mathrm{X}^{*}$ & $X^{*}$ & $\mathrm{X}^{*}$ & - & $\mathrm{X}^{*}$ & $\mathrm{X}$ & $\mathrm{X}$ & $\mathrm{X}$ & $\mathrm{X}$ & $\mathrm{X}$ & $\mathrm{X}^{*}$ & $\mathrm{X}$ & $\mathrm{X}$ & $X^{*}$ \\
\hline S. quebeckensis (Walley) & - & $X$ & $X^{*}$ & - & $\mathrm{X}$ & $X^{*}$ & $\mathrm{X}^{*}$ & - & $\mathrm{X}$ & - & - & - & - & - \\
\hline S. signata (Fieber) & $\mathrm{X}$ & $\mathrm{X}$ & $\mathrm{X}^{*}$ & $\mathrm{X}$ & $\mathrm{X}$ & $\mathrm{X}$ & $\mathrm{X}$ & $\mathrm{X}$ & $\mathrm{X}$ & $\mathrm{X}$ & $\mathrm{X}^{\star}$ & $\mathrm{X}$ & $\mathrm{X}$ & $\mathrm{X}$ \\
\hline S. solensis (Hungerford) & $X^{9}$ & $x$ & $X^{*}$ & - & $\mathrm{X}$ & - & $x$ & $x$ & $x$ & $x$ & $X^{*}$ & $x$ & - & - \\
\hline S. stigmatica (Fieber) & $-{ }^{7}$ & $X^{*}$ & - & $X^{*}$ & $X^{*}$ & - & $X^{*}$ & - & - & - & - & - & - & - \\
\hline S. transfigurata (Walley) & $\mathrm{X}^{\star}$ & $\mathrm{X}^{*}$ & - & $x$ & - & - & - & $\mathrm{X}$ & $x$ & - & - & - & - & - \\
\hline S. trilineata (Provancher) & $X^{*}$ & $X^{*}$ & $X^{*}$ & $X^{*}$ & - & - & - & $x$ & $x$ & $x$ & $X^{*}$ & - & $x$ & $X^{*}$ \\
\hline S. variabilis (Hungerford) & $\mathrm{X}^{\star}$ & $\mathrm{X}^{*}$ & $X^{*}$ & - & $\mathrm{X}$ & $X^{*}$ & $\mathrm{X}^{\star}$ & $\mathrm{X}$ & $x$ & $x$ & $\mathrm{X}^{\star}$ & $\mathrm{X}$ & - & - \\
\hline S. virginiensis Hungerford & - & $x$ & - & - & $\mathrm{X}^{*}$ & $X^{*}$ & $\mathrm{X}^{*}$ & - & - & - & - & - & - & - \\
\hline S. zimmermanni (Fieber) & $x$ & - & - & $x$ & $X$ & - & $X$ & - & - & - & - & - & - & - \\
\hline Trichocorixa borealis Sailer & - & - & $X^{*}$ & - & - & - & - & $\mathrm{X}$ & $x$ & - & - & - & - & - \\
\hline T. calva (Say) & - & $X^{*}$ & $X^{*}$ & - & $\mathrm{X}^{\star}$ & $\mathrm{X}^{*}$ & $\mathrm{X}$ & $\mathrm{X}$ & - & - & - & - & - & - \\
\hline T. louisianae Jaczewski & - & $X$ & - & $\mathrm{X}$ & $\mathrm{X}$ & $X^{*}$ & $X$ & - & - & - & $X^{10}$ & - & - & - \\
\hline T. macroceps (Kirkaldy) & - & $\mathrm{X}$ & - & $\mathrm{X}^{*}$ & $\mathrm{X}^{\star}$ & $X^{*}$ & $\mathrm{X}$ & $\mathrm{X}^{*}$ & - & - & - & - & - & - \\
\hline T. sexcincta (Campion) & $X^{*}$ & $\mathrm{X}^{*}$ & $\mathrm{X}^{*}$ & $\mathrm{X}^{11}$ & $\mathrm{X}$ & $X^{*}$ & $X^{11}$ & $X^{11}$ & $X^{11}$ & - & $\mathrm{X}$ & $\mathrm{X}^{*}$ & $\mathrm{X}$ & $\mathrm{X}^{*}$ \\
\hline T. verticalis verticalis (Fieber) ${ }^{12}$ & $\mathrm{X}$ & $\mathrm{X}$ & - & $\mathrm{X}$ & $\mathrm{X}$ & $\mathrm{X}$ & $\mathrm{X}$ & - & $\mathrm{X}^{13}$ & $\mathrm{X}^{*}$ & $\mathrm{X}^{\star}$ & $\mathrm{X}^{*}$ & $\mathrm{X}^{13}$ & $X^{*}$ \\
\hline
\end{tabular}

1 The species of Dasycorixa and Glaenocorisa are mainly pelagic plankton feeders, and are not necessarily caught in sampling done from shorelines. Therefore, the records of these species are rather scattered.

${ }^{2}$ As Dasycorixa sp. in Larson and Colbo (1983).

${ }^{3}$ Reporded for QUE by Hungerford (1948), Lansbury (1955), Henry and Froeschner (1992) and for NFL by Larson and Colbo (1983) with the synonymic name G. quadrata Walley (synonymy established by Ossiannilsson (1960)). Generally considered as a subspecies of G. propinqua (Fieber), but raised to species rank by Jansson (2000) because the two are sympatric in Scotland (Jansson 1986) and in northern Finland (unpublished data). Also Ossiannilsson (in Lindroth and Ball (1969)) took the same attitude although he did not officially propose $G$. cavifrons as a separate species.

${ }^{4}$ Reported for NY by Hungerford (1948), but not by Polhemus et al. (1988).

${ }^{5}$ Incorrect emendations as H. harrisi and H. kennicotti by Polhemus et al. (1988), see the Code, Art. 33 (d).

${ }^{6}$ Hungerford (1948) reported also one female from RI (also listed in Polhemus et al. (1988)), but this is an obvious misidentification and the specimen could not be located in the USNM (Coll. C. F. Baker).

${ }^{7}$ Specimen illustrated by Hungerford (1948) as closest to S. stigmatica in Plate XCIV from NY, Putnam, seems to be in fact $S$. gordita.

${ }^{8}$ Reported for NFL by Lansbury (1955) and Larson \& Colbo (1983), but not by Polhemus et al. (1988).

${ }^{9}$ Reported for ME by Hungerford (1948), but not by Polhemus et al. (1988).

${ }^{10}$ A record from the Sable Island!

${ }^{11}$ As T. naias (Kirkaldy) by Sailer (1948) and Lansbury (1955).

${ }^{12}$ Reported by Polhemus et al. (1988) as T. verticalis (Fieber) for ME, NH, MA, CT, RI, NY and QUE, as T. verticalis fenestrata (Walley) for QUE, as $T$. verticalis sellaris (Abbott) for $\mathrm{ME}, \mathrm{NH}, \mathrm{MA}, \mathrm{RI}$, and $\mathrm{NY}$, and as $T$ verticalis verticalis (Fieber) for NY, thus causing unnecessary confusion and incorrectly interpreting Sailer (1948) who clearly indicated that $T$. verticalis var. sellaris (Abbott, 1913) has no nomenclatural status.

${ }^{13}$ As $T$. verticalis fenestrata (Walley).

Many of these species are lacking from adjacent states or provinces, but can be expected to be found from them with more intensive collecting. Also, in this report I am only listing the states and the provinces, and the exact locality records will be shown in the maps in the forthcoming revision.

In the present paper I am reporting also those states and provinces for which I do not necessar- ily have verified records. However, the record by Lansbury (1955) for Arctocorisa sutilis (Uhler) for Quebec (Great Whale River) is omitted because it is most likely a misidentification or mislabeling. The verified records for this species are limited to the Rocky Mountains and a few localities in northern Canada, the most eastern being from Saskatchewan. 
In this context I also establish a new synonymy Trichocorixa verticalis fenestrata (Walley, 1930), which is considered a synonym of the nominate subspecies. There is a clear transitional zone from typical $T$. verticalis verticalis (Fieber, 1851) to $T$. verticalis "fenestrata" i.e. a cline in which the most northern populations differ to some degree from the southern populations.

Acknowledgments. I wish to thank the following colleagues for material sent to me for identification: Dr. Ross T. Bell (Burlington, VT), Dr. Robert W. Brooks (Lawrence, KS), Dr. Donald S. Chandler (Durham, NH), Mme M. Coulloudon (Montreal, QUE), Dr. C. Ewing (Halifax, NS), Dr. K. E. Gibbs (Orono, ME), Dr. K. G. A. Hamilton (Canadian National Collection, Ottawa, ONT), Dr. D. J. Larson (St. John's, NFL), Dr. J. E. O’Donnell (Storrs, CT), Dr. R. J. Pupedis (New Haven, CT), the late Dr. C. V. Reichard (Providence, RI), Dr. C. Schaefer (Storrs, CT), Dr. R. T. Schuh (American Museum of Natural History, NY), Dr. G. G. E. Scudder (Vancouver, B.C), and Dr. G. B. Wiggins (Toronto, ONT).

\section{References}

Henry, T. J. \& Froeschner, R. C. 1992: Corrections and additions to the "Catalog of the Heteroptera, or true bugs, of Canada and the continental United States". Proc. Entomol. Soc. Washington 94: 263-272.

Hungerford, H. B. (ed.) 1948: The Corixidae of the West- ern Hemisphere (Hemiptera). — Univ. Kansas Sci. Bull. 32: $1-827$.

Jansson, A. 1986: The Corixidae (Heteroptera) of Europe and some adjacent regions. - Acta Entomol. Fennica 47: 1-94.

Jansson, A. 2000: Interesting collection of Corixidae (Heteroptera) from a fish pond. — Entomol. Fennica 11: $183-184$.

Lansbury, I. 1955: Distributional records of North American Corixidae (Hemiptera: Heteroptera). — Canadian Entomol. 87: 474-481.

Larson, D. J. \& Colbo, M. H. 1983: The aquatic insects: biogeographic considerations. - In: South, G. R. (ed.), Biogeography and Ecology of the Island of Newfoundland. Dr. W. Junk Publishers, The Hague. pp. 593-677.

Lindroth, C. H. \& Ball, G. E. 1969: An annotated list of invertebrates of the Kodiak Island Refugium. - In: Karlstrom, T. N. \& Ball, G. E. (eds.), The Kodiak Island Refugium: Its Geology, Flora, Fauna and History. Boreal Institute, University of Alberta, Ryerson Press. pp. 22-155.

Ossiannilsson, F. 1960: On Glaenocorisa cavifrons Thoms. (Hem., Heteropt., Corix.). — Opusc. Entomol.25:170172.

Polhemus, J. T., Froeschner, R. C. \& Polhemus, D. A. 1988: Family Corixidae Leach, 1815. — In: Henry, T. J. and Froeschener R. C. (eds.), Catalog of the Heteroptera, or true bugs, of Canada and the continental United States. E. J. Brill, Leiden, New York. pp. 93-118.

Sailer, R. I. 1948: The genus Trichocorixa (Corixidae, Hemiptera). - In: Hungerford, H. B. (ed.), The Corixidae of the Western Hemisphere, Hemiptera. Univ. Kansas Sci. Bull. 32, pp. 289-407. 